\title{
What Do Teachers Desire in Music Digital Textbook for Leaner-Centered Curriculum?
}

\author{
Sahoon H. Kim \\ College of Education, Hankuk University of Foreign Studies, Seoul, South Korea \\ Email: kimsh@hufs.ac.kr
}

Received November 2014

\begin{abstract}
We are living in the 21st century that requires us to know how to integrate paper based curricula into interactive media. The purpose of the study is to provide exemplary cases that music teachers hope for their music classes through using Digital Textbook. For this purpose, two times of interviews with ten music teachers in South Korea were conducted. Case study and grounded theory methodology were adopted as a tool for analyzing the data. As a result, the teachers' desires for Digital Textbook were categorized into two perspectives: learner-centered learning, and collaborative learning \& connection with open sources.
\end{abstract}

\section{Keywords}

Digital Textbook, Music Curriculum, Learner-Centered Learning, Collaborative Learning

\section{Introduction}

Five years ago, South Korea mapped out a plan to transform its education system into the world's most cutting-edge. The country would turn itself into a knowledge powerhouse, one government report declared, breeding students equipped for the future. These students would have little use for the bulky textbooks familiar to their parents. Their textbooks would be digital, accessible on any screen of their choosing. Their backpacks would be much lighter [1].

What changes digital textbooks (DT) can bring to the traditional classroom context as well as how to integrate paper based curricula into this interactive media available device has been a hot issue for researchers and educators throughout the world. Especially since 1997, as one of the leading countries in the field, the government of South Korea has attempted to develop DT [2]. Even though, at the current stage, it is difficult to say whether it is a success or failure, one cannot disagree that it is worthwhile to study the digital textbook [3]. Particularly because of its affordances for interactive learning, researchers strongly argue that it would provide a great benefit for the music classroom.

The purpose of the study was to explore and suggest features that music teachers want in digital textbooks for their music classes. For this purpose, this article firstly summarizes how the use of DT was implemented in Ko- 
rea. By drawing out the opportunities and challenges of DT in general, this study attempted to shed light on how to construct DT for music curricula. As a data gathering process, this study interviewed ten music teachers to ask them what properties they hoped to develop in DT for music curricula.

\section{Development of Digital Textbooks in Korea}

An ambitious digitalized textbook project has been ongoing since 1997 in Korea. At that time, called electronic textbooks, digital textbooks were shown to learners in the shape of digitalized material such as CD-ROM, WEB, and PDA through scanning traditional paper textbooks. With the development of technology, the efforts to construct DT came to include more functions such as searching and multimedia. In 2007, single chapters from 9 subjects in 5th grade elementary textbooks were developed into DT. In the following year, 6 subjects (Korean, English, mathematics, social studies, science, and music) for 5th graders were developed in a Windows-based platform. In addition, 6th grade Korean, social studies, science, and mathematics were developed in a Linux-based platform. In 2009, 2 subjects for 4th grade and middle school were developed into DT [4].

More recently, the Korean government announced a plan to innovate education via the SMART Learning Initiative. SMART stands for Self-directed, Motivated, Adaptive, Resource Enriched, and Technology Embedded. There are five major elements in the plan. First, develop digital textbooks, and have students access educational

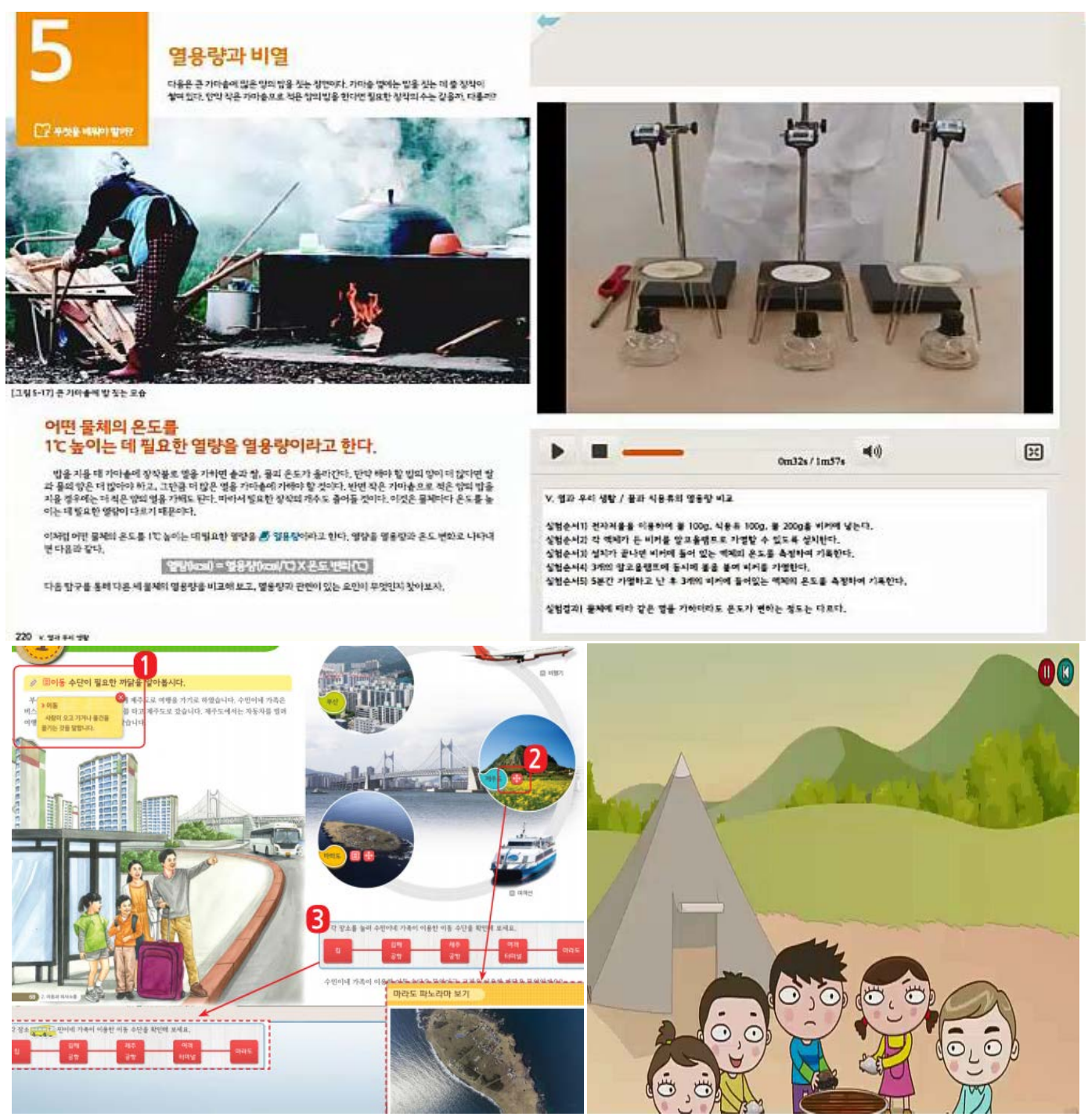

Figure 1. Screen captures of DT developed in Korea (Captured from book. edunet.net, 2014. 10. 1). 
contents via smart-phones and other devices. Second, stimulate the use of on-line classes and establish an online evaluation system. Third, build a safe and accessible environment for the use of educational materials. Fourth, enhance the practical competencies of teachers preparing to use SMART education. Fifth, equip infrastructures for Cloud services, including developing a SMART platform and the distribution of Cloud PCs. The Ministry of Education plans to develop DT for all subjects for elementary school students by 2014, and for middle and high school students by 2015 (see Table 1). However, paper textbooks will be used simultaneously. The estimated budget allocation for DT development is 45.5 billion Won (3.3 billion Yen) [5]. Reforming education via DT could be seen in Japan and Malaysia, too.

In sum, "digital textbook" is different from "electronic textbook" (e-textbook). Comparing to the electronic textbook, referred to as an e-textbook, which is digitalized by just scanning a textbook, DT includes functions such as searching, navigating, and multimedia. In a study by [6], he explained that the DT platform is an XML base, which follows worldwide standards and supports resource management by associating XML elements with Uniform Resource Identifiers (URIs). Once students sign up at the main server, contents of DT are downloaded and installed in students' tablet PCs. Students can log-in and use the DT in class. Any notes and personal data are saved on the server.

Figure 2 shows exemplary digital textbook platform. The official DT home page is http://www.dtbook.kr.

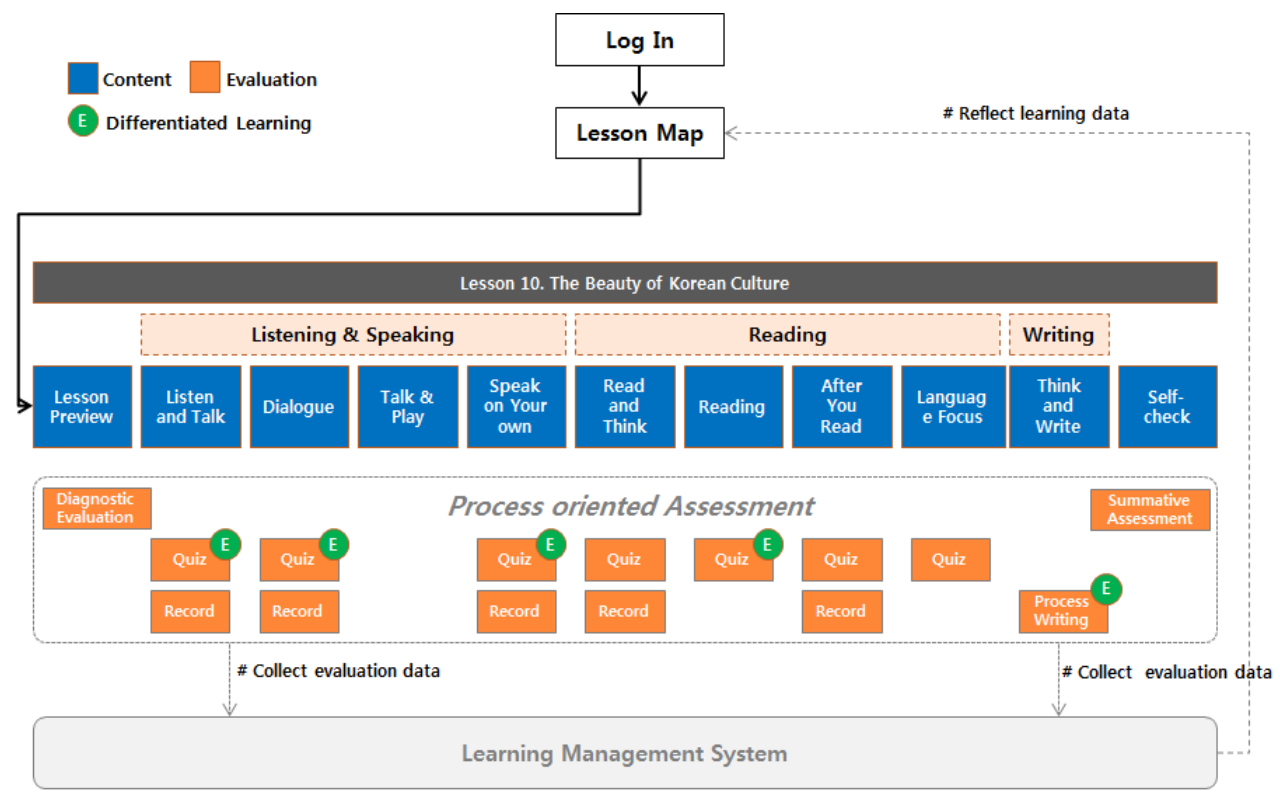

Figure 2. Digital textbook platform [7].

Table 1. Digital textbooks developed in Korea (Ministry of Education, 2011).

\begin{tabular}{cccc}
\hline Year & Grade & & Developed Subjects \\
\hline 2008 & 5 & Korean, English, mathematics, social studies, science, music \\
& Elementary & Korean, mathematics, social studies, science \\
\hline 2009 & Elementary & $3-6$ & English(4 kinds in different level) \\
& Middle & 1 & social studies, science \\
\hline 2010 & Elementary & $5-6$ & Revision of social studies and science \\
\hline & Elementary & $5-6$ & Revision of Korean and mathematics
\end{tabular}




\section{Methodology}

To explore the features of DT desirable for music subjects, we conducted two times of interviews with ten music teachers in South Korea. Case study methodology and some skills from grounded theory methodology was adopted as a tool for analyzing the data (e.g., open coding, memoing, and constant comparison) [8]. Grounded theory is a methodology developed by Glaser and Strauss in the mid-1960s while the two worked together on an extended qualitative research project on palliative care in hospitals [9]. Grounded Theory is to examine the daily life of groups of people by investigating executive abilities, behaviors, conviction, and attitude of individuals or groups of individuals. The methodology of Grounded theory involves building theory from data by way of a specific set of methods. In other words, it aims to theoretically explain phenomenon by providing models rather than describe in a specific way. Following the process, this study focused on making a categorization from narratives of participants.

The first data for research were from casual interviews, and the second were from in-depth interviews. The questions were made in advance using articles about DT and previous interviews for structured interviews. The conversation with music teacher during the break or lunch time was also used. After gathering the data, the concepts, patterns, and core categories were analyzed.

As a second step of the analysis, the researcher developed relevant categories and patterns through the qualitative analyzing tool, Nvivo 8. Analysis was conducted independently by the authors, noting emerging themes. As a next step, relevant issues such as duplicated categories and mismatches were discussed. Through the discussion, the data were triangulated to check the emerging themes. The results were constructed after the researchers mutually agreed on every conflicting theme.

\section{Result}

While interviewing participants, we could draw somewhat significant meanings from the data. Teachers' hopes for DT are not just centered on changing the learning material but also include the learning contexts available through the tool.

The domain of the recently revised "2009 Revised Curriculum for Music" is categorized into "expression, appreciation, and music in everyday life". Therefore, it is necessary that the music curriculum is developed in a way that expression (singing, instrumental music, and music creation), appreciation, and music in everyday life could be well embodied in its design and technology (DT). Classified into two types of learning, student-centered and collaborative, the information on each type can be proposed as the following.

\subsection{Learner-Centered Learning with DT}

Since music is a temporal art, activities involving the direct listening of various kinds of music during class would be critical. However, students would also need a diversity of visual information, such as the corresponding musical score and lyrics, in order to truly enjoy the educational experience of listening to music. In this respect, in student-centered learning, the most significant components would be presenting a number of visual resources, numerous kinds of listening activities, etc., through the DT.

One of the elements most frequently found from the data was "like a Garage Band". Music teachers hoped that digital textbooks for music should bring learners' participation, not a teacher-centered class. For the purpose it seemed that they essentially think that the functions of Garage Band might be able to make this context for their students. Then, the use of "Garage Band like functions" is categorized again according to the purpose of using those functions.

\subsubsection{Visualization of the Notes and Lyrics}

Major parts of our data showed that teachers want cutting-edge technology for DT that might bring visualization to their music class. In DT, the book should have the functions of playing the song along with visualized notes and scores. By including this function they said that students easily could learn scales, notes, and pitch in embedded contexts. Although existing printed textbooks carry musical scores, not all of them are included due to the limited number of pages, and the presentation of musical scores related to music appreciation is lacking in particular. Thus, the DT should include various scores such as pictorial music scores, bar musical notes, and sheet music, and especially scores and lyrics that can be utilized during music listening activities. This DT, in 
addition to a variety of musical scores, is expected to be beneficial for student-centered learning with the "visual information that allows students to identify where the exact note is according to the music". In detail, if there is a visual representation of which note the music performed at the moment corresponds to, students will be able to learn music by themselves. Figure 3 is a screen capture of the garage band, which shows desirable function music teacher wanted for DT.

\subsubsection{Listening with Various Musical Instruments}

Another function teachers wanted through "Garage Band" was allowing learners to listen to various musical instruments and mix them together (see Figure 4). Music teachers hoped learners could enjoy music in the form of the sound of various instruments and how their sound could be mixed and harmonize. The experience of Garage Band was one interface they hoped to include in the textbook. By making DT in this way, teachers expected learners could enjoy the music with self-directed motivation.

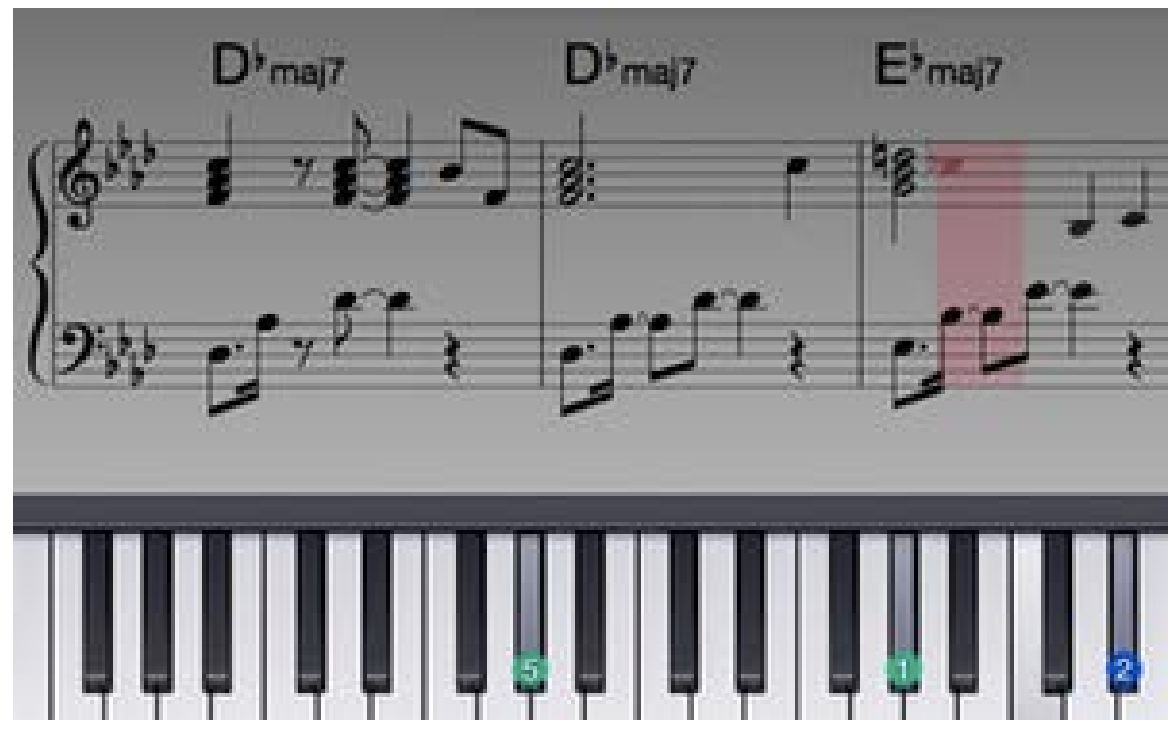

Figure 3. A screen capture of a function music teachers wanted for DT (apple.com).

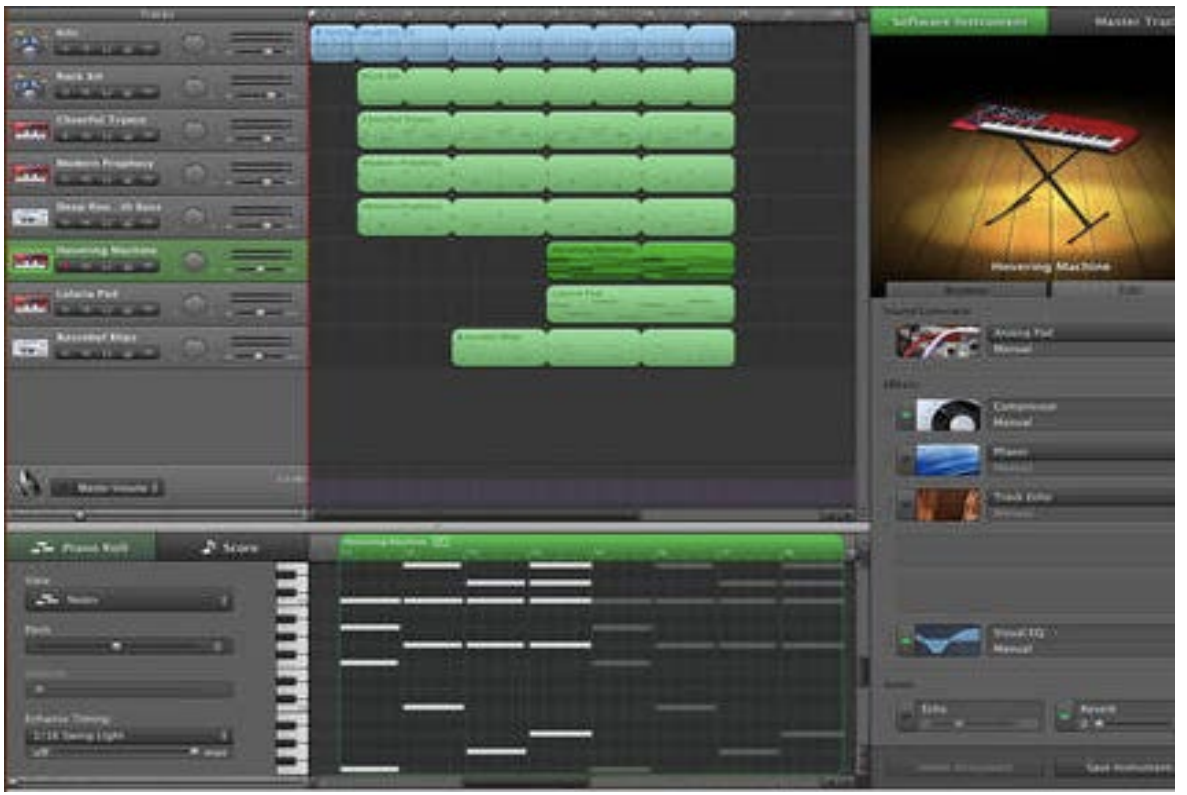

Figure 4. A screen capture of a function music teachers wanted for DT (apple.com). 
In regards to printed textbooks, separate CDs are available since music cannot be incorporated into them. However, there are difficulties in mixing or changing existing music, as well as a limitation in involving diverse kinds of music. On the other hand, the DT is equipped with various sound sources which allow the students to listen to different instrumental sounds and search for them. Another useful aspect is that varied tones can be created by combining several kinds of instruments. The DT is also valuable in that it includes numerous types of music from different time periods and cultures. For instance, students can compare musical pieces of a certain period, or listen firsthand to music of different cultures that they had only encountered through texts.

\subsection{Collaborative Learning through Connection with Open Source}

Another category captured from the data was collaborative learning. Teachers' hopes for a digital textbook for their music class was not just to supply digitalized and learner-centered environments, but also to provide a learner environment allowing their students to collaborate with their classmates and to network-link users from different contexts. Since activities concerning experiencing and performing are important in music, learning in a collaborative manner with fellow students would be a vital element of the learning experience.

"I think using digital textbook should show capability to make classmate to collaborate. It can be shown in composition class, harmony class, or any kind of music class. Then, I think making on-line and collaborating with other countries' students also making student interest” (jihyun, music teacher)

In fact, four of our participants from second interview showed strong trust in having the connection with open sources available with the digital textbook. Because there already exist well-made open sources for learning in YouTube and copious on-line learning sites, teachers perceived making a connection with those open sources as one of the vital elements for the success of the future digital textbook. By incorporating those sources, teachers hoped the digital textbook might bring changes to the traditional teacher-centered and teaching-focused music class, turning it into learner-centered and learning with self-directed motivation.

\subsubsection{Collaborative Music Activities through Student Interaction in the Classroom}

Collaborative learning through students' interactions would be especially useful when engaging in "creative” activities. Such learning can be carried out by using the DT and music composition software (finale, encore, etc.) presented in it. Students could be in charge of one musical element each; for example, Student A can work on the rhythm, Student B can be in charge of the chord, and Student C could take care of the melody, to create a musical piece. Through such collaborative process, students will be able to listen to each musical part as well as harmonize and cooperate with one another.

\subsubsection{Collaborative Musical Activities beyond the Classroom, in Connection with Other Learning Environments}

Going beyond the classroom, diverse kinds of collaborative learning can take place by connecting them with open spaces and materials. This could be linked to "music in everyday life", which is emphasized in the music curriculum. Students would be able to carry out musical activities by utilizing various spaces and materials that help make music a part of daily life. In particular, it would be useful for learning music through various scenes or live performances, as well as for learning about music from different cultures and regions.

\section{Conclusions}

We are living in the 21st century that requires us to know how to integrate paper based curricula into interactive media. DT is not a fantasy. It is an educational tool available in today's educational field. DT is moving from simply e-textbooks to digital learner-centered media that encourage learners' activity. The purpose of the study is to provide exemplary cases that music teachers hope for their music classes through using Digital Textbook. For this purpose, interviews with ten music teachers in South Korea were conducted.

With the analysis of teachers' perception of DT, the researcher suggested big pictures incorporating needs of the field. First, this study illuminated that the appropriate nature of DT was on the function of visualization, which eventually might bring leaners to engage in their class. They expected that instructional materials should support students' cognitive development. They believed that learner-centered learning in music could be facilitated through visual assistances. Indeed, participants hoped learners could enjoy music in the form of the sound of various instruments and how their sound could be mixed and harmonize. They also required DT to be ex- 
tended to the area which might incorporate open sources. By using open sources, they expected learners to collaborate with their colleagues.

The development and implementation of curriculum should include voices of teachers [10]. Especially, for art education, the prescriptive development models, such as one of Tyler's [11] and Taba's [12], have limitations because their focus is not on students' activity. According to Eisner [10], learners' goal should be continuously revised throughout classes according to their interactions.

Accordingly, in music education, use of DT would be expected to change a lot of features in the current classroom. Through the study, we explored what teachers hope for in the near future from the digital textbook. They stressed that DT should have functions that will direct learners into directly experiencing the joy of music. I also expect that through examining teachers' perceptions for DT, we could bring about better results for DT projects, which have had a lot of problems occasioned by a top-down approach, without considering teachers and students.

\section{Acknowledgements}

This work was supported by Hankuk University of Foreign Studies Research Fund of 2014.

\section{References}

[1] Harlan, C. In South Korean Classrooms, Digital Textbook Revolution Meets Some Resistance. Washington Post. http://www.washingtonpost.com/world/asia_pacific/in-south-korean-classrooms-digital-textbook-revolution-meets-so me-resistance/2012/03/21/gIQAxiNGYS_story_1.html

[2] Byun, H., Yoo, K., Yoo, J., Choi, J. and Park, S. (2005) 2005 Study on Standardization of Electronic Textbook Development. KERIS, Research Report CR 2005-22.

[3] Kim, H.S., Lee, Y.-A., Lee, D.Y. and Leem, J. (2013) Development of Teaching and Learning Models for the Effective Use of Paper and Digital Textbooks. KICE, Research Report RRT 2013-2.

[4] Noh, K., Kim, B. and Lee, W. (2011) 2010 Digital Textbook Effectiveness Research. KERIS, Research Report CR 2011-1.

[5] Ministry of Education (2011) The Pathway to Great Human Resource Nation: SMART Education Strategy Implementation Plan. Ministry of Education. Unpublished document.

[6] Song, Y. and Byun, H. (2010) Status of Digital Textbooks and Its Challenges. Review of Korean Society of Internet Information, 28, 58-63.

[7] Joo, H., Ahn, J., Ga, E. and Nam, C. (2013) A Study on the Development of Digital Textbooks: Designing a Prototype for English Digital Textbooks. KICE, Research Report RRT 2013-1-1.

[8] Glaser, B.G. and Strauss, A.L. (1967) The Discovery of Grounded Theory. Aldinede Gruyter, New York.

[9] Glaser, B.G. and Strauss, A.L. (1965) Awareness of Dying. Aldine de Gruyter, New York.

[10] Eisner, E.W. (1979) The Educational Imagination. Macmillan, New York.

[11] Tyler, R.W. (1949) Education 206: Basic Principles of Curriculum and Instruction. University of Chicago Press, Chicago.

[12] Taba, H. (1962) Curriculum Development: Theory and Practice. Harcourt, Brace \& Jovanovich, New York. 